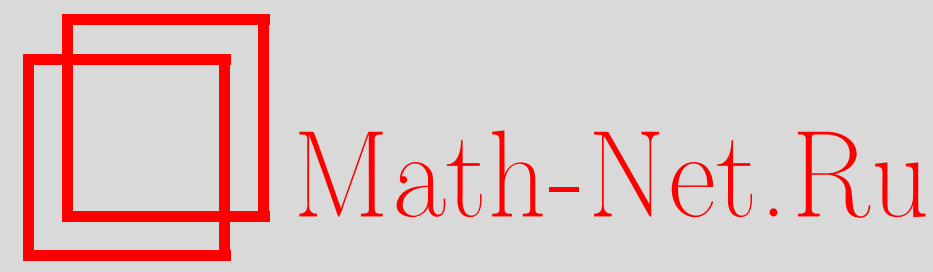

И. В. Соболев, Об одной серии бирационально жестких многообразий с пучком гиперповерхностей Фано, Матем. сб., 2001, том 192, номер 10, 123-130

DOI: https://doi.org/10.4213/sm605

Использование Общероссийского математического портала Math-Net.Ru подразумевает, что вы прочитали и согласны с пользовательским соглашением http://www . mathnet.ru/rus/agreement

Параметры загрузки:

IP: 35.174 .16 .151

26 апреля 2023 г., 09:28:35 


\title{
И.В. Соболев \\ Об одной серии бирационально жестких многообразий с пучком гиперповерхностей Фано
}

\author{
В работе доказьвается, что общий дивизор бистепени $(2, M)$ в $\mathbb{P}^{1} \times \mathbb{P}^{M}$ при \\ $M \geqslant 4$ является бирационально жестким многообразием и группа его бирацио- \\ нальных автоморфизмов состоит из двух элементов. \\ Библиограбфия: 11 названий.
}

\section{§1. Введение}

В настоящей работе исследуются бирациональные свойства многообразия $V$, которое задается в $\mathbb{P}^{1} \times \mathbb{P}^{M}$ уравнением

$$
A\left(x_{0}, \ldots, x_{M}\right) u^{2}+B\left(x_{0}, \ldots, x_{M}\right) u v+C\left(x_{0}, \ldots, x_{M}\right) v^{2}=0
$$

где $A, B, C$ - однородные многочлены степени $M \geqslant 4$.

Геометрия многообразия $V$ представляется интересной в силу нескольких причин. Многие важные результаты, относящиеся к проблеме рациональности многомерных алгебраических многообразий, были получены с помощью метода максимальных особенностей, созданного В. А. Исковских и Ю. И. Маниным (см. [1]) и усовершенствованного А.В. Пухликовым. Нерациональность многообразий, к которым применяется этот метод, является следствием их бирациональной (сверх)жесткости. В работах [2], [3] доказано, что при $m \geqslant 3$ и $M \geqslant 4$ обшее многообразие $V_{m}$, которое является дивизором бистепени $(m, M)$ в $\mathbb{P}^{1} \times \mathbb{P}^{M}$, бирационально сверхжестко и обладает единственной структурой Фано-расслоения. С другой стороны, $V_{1}$ рационально при всех $M$. Поэтому на примере многообразия $V=V_{2}$ можно проследить, как происходит переход от сверхжесткости к рациональности. Отметим, что нерациональность многообразия $V_{2}$ при $M=3$ доказана в работе [4] другим методом.

В настоящее время очень важньм является также вопрос о границах применимости метода максимальных особенностей. В серии работ [2], [3], [5] установлена бирациональная жесткость некоторых классов Фано-расслоений, удовлетворяющих так называемому $K^{2}$-условию. Для определения наибольшей возможной области применения метода максимальных особенностей полезно рассматривать примеры многообразий, для которых это условие не выполнено. Такие примеры исследуются в работах [6], [7]. Многообразие $V$ доставляет еше один пример подобного рода.

Автор благодарит А.В. Пухликова за постановку и обсуждение проблемы.

\section{§2. Формулировка результата}

Прежде чем сформулировать наш основной результат, дадим некоторые определения и напомним основные понятия метода максимальных особенностей.

Работа вьполнена при поддержке Российского фонда фундаментальных исследований (грант № 99-01-01132) и фонда INTAS-OPEN (грант № 97/2072). 
Фиксируем отображения $\pi: V \rightarrow \mathbb{P}^{1}, \sigma: V \rightarrow \mathbb{P}^{M}$, которые являются ограничениями проекций $\mathbb{P}^{1} \times \mathbb{P}^{M}$ на первый и второй множители соответственно. Проекция $\pi$ задает структуру расслоения $\Phi$ ано на $V$.

Пусть $F$ - гиперповерхность степени $M$ в $\mathbb{P}^{M}$. Пусть $p \in F-$ произвольная точка и в аффинной системе координат с центром в $p$ уравнение $F$ имеет вид $q_{1}+\cdots+q_{M}=0$, где $q_{i}$ - однородный многочлен степени $i$.

ОПРЕДЕЛЕНИЕ 1. Гладкую точку $p \in F$ назовем регулярной, если последовательность $\left(q_{1}, \ldots, q_{M-1}\right)$ регулярна. Будем говорить, что особая точка $p \in F p e z y$ лярна, если последовательность $\left(q_{2}, \ldots, q_{M}\right)$ регулярна, квадрика в $\mathbb{P}^{M-1}$, заданная уравнением $q_{2}=0$, является гладкой и система уравнений $q_{2}=\cdots=q_{M}=0$ определяет в $\mathbb{P}^{M-1}$ ровно $M$ ! различных точек, каждые $M$ из которых линейно независимы.

ОПРЕДЕЛЕНИЕ 2. Многообразие $V$ будем назьвать регулярным, если

1) для любого $t \in \mathbb{P}^{1}$ гиперповерхность $\pi^{-1}(t)$ является $\mathbb{Q}$-факториальным многообразием, каждая точка которого регулярна в смысле определения 1 ,

2) многообразие $W \subset \mathbb{P}^{M}$, заданное уравнениями $A=B=C=0$, является гладким неприводимым многообразием размерности $M-3$.

ПРЕДЛОЖЕНИЕ 1. Общее многообразие $V$ является регулярным.

ДокАЗАТЕльство. Условие 2), очевидно, выполняется для общего $V$. С помощью счета констант в работе [3] доказано, что условие 1) тоже выполнено.

ОПРЕДЕЛЕНИЕ 3. Порогом канонического присоединения дивизора $D$ на $\Phi$ ано-расслоении $\rho: V \rightarrow S$ называется число

$$
c(D, V)=\sup \left\{\frac{b}{a}: a, b \in \mathbb{Z}_{+} \backslash\{0\},\left|a D+b K_{V}\right| \neq \varnothing\right\} .
$$

ОПРЕДЕЛЕниЕ 4. Фано-расслоение $\rho: V \rightarrow S$ называется бирационально жестким, если для любого Фано-расслоения $\rho^{\prime}: V^{\prime} \rightarrow S^{\prime}, \operatorname{dim} V^{\prime}=\operatorname{dim} V$, бирационального отображения $\chi: V \rightarrow V^{\prime}$ и линейной системы $\left|D^{\prime}\right|$ на $V^{\prime}$, свободной в коразмерности 1 , существует бирациональный автоморфизм $\chi^{\prime}$ многообразия $V$ такой, что $c(D, V) \leqslant c\left(D^{\prime}, V^{\prime}\right)$, где $|D|=\left(\chi \circ \chi^{\prime}\right)_{*}^{-1}\left|D^{\prime}\right|$.

Многообразие $V$ имеет вторую структуру Фано-расслоения. Рассмотрим отображение $\tau: V \rightarrow V$, которое переставляет точки в слоях $\sigma$. Оно определено вне подмножества $\sigma^{-1}(W)$ и является бирациональной инволюцией. Вторая структура получается действием $\tau$ на первую.

Мы докажем следующий результат:

Теорема. Регулярное многообразие $V$ бирационально жсестко при всех $M \geqslant 4$. Группа $\operatorname{Bir} V$ для общего $V$ изоморфна $\mathbb{Z}_{2}$ и порождается $\tau$.

СледСтВИЕ. 1) Многообразие $V$ обладает лишь двумя структурами Фанорасслоения; $V$ не имеет структур расслоения на многообразия Фано, размерность которых меньше $M-1$.

2) Многообразие $V$ не рачионально.

\section{§3. Максимальные особенности}

Пусть теперь $\rho: V^{\prime} \rightarrow S$ - некоторое Фано-расслоение, $\left|D^{\prime}\right|$ - линейная система на $V^{\prime}$ и $\chi: V \rightarrow V^{\prime}-$ бирациональное отображение, для которого $c(D, V)>$ $c\left(D^{\prime}, V^{\prime}\right)$. Пусть $|D|=\chi_{*}^{-1}\left(\left|D^{\prime}\right|\right)$. Группа Рic $V$ порождается каноническим классом $K_{V}$ и классом $F$ слоя проекции $\pi$, поэтому $D \sim-n K_{V}+l F$, где $n \geqslant 0$. 
ОПреДЕЛЕниЕ 5. Дискретное нормирование $\nu$ поля рациональных функций многообразия $V$ называется максимальной особенностью бирационального отображения $\chi$, если выполняется неравенство Нётера-Фано-Исковских $e(\nu)=$ $\nu(|\chi|)-n K(\nu, V)>0$, где $K(\nu, V)$ - каноническая кратность $\nu$ по отношению к $V$ (см. [8]).

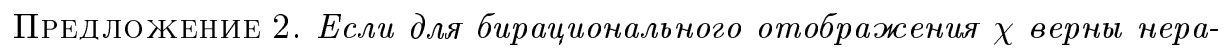
венства $c(D, V)>c\left(D^{\prime}, V^{\prime}\right), l \geqslant 0$, то иентры всех максимальных особенностей $\chi$ лежат в слоях $\pi$ и существует конечное множество максимальных особенностей М такое, что выполнено следующее неравенство:

$$
\sum_{t \in \mathbb{P}^{1}} \max _{\left\{\nu \in \mathscr{M}: \operatorname{centre}(\nu) \subset F_{t}\right\}} \frac{e(\nu)}{\nu\left(F_{t}\right)}>l,
$$

əде $F_{t}=\pi^{-1}(t)$.

Доказательство см. в [3].

Пусть $D_{i} \in|D|$ - общие дивизоры и $Z=\left(D_{1} \circ D_{2}\right)$ - цикл коразмерности 2 , $Z=Z^{v}+Z^{h}$, где $Z^{h}$ накрывает $\mathbb{P}^{1}$, а компоненты $Z^{v}$ лежат в слоях проекции $\pi$ :

$$
Z=Z^{h}+\sum_{t \in \mathbb{P}^{1}} Z_{t}^{v}, \quad \operatorname{supp} Z_{t}^{v} \subset F_{t} .
$$

Под степенью цикла $Z_{t}^{v}$ мы понимаем его степень как подмногообразия проективного пространства. Степенью цикла $Z^{h}$ будем назьвать степень его пересечения с общим слоем $\pi$. Степень цикла $Y$ обозначим через $\operatorname{deg} Y$.

Группа $\mathrm{Pic} \mathbb{P}^{1} \times \mathbb{P}^{M}$ порождена классом тавтологического пучка $H$ и классом $G$ слоя проекции на $\mathbb{P}^{1}$. Пусть $H_{V}=\left.H\right|_{V}, F=\left.G\right|_{V}$. Имеем $K_{V}=-H_{V}, H_{V}^{M}=2$, $H_{V}^{M-1} F=M, F^{2}=0$. Из равенства (1) получим

$$
\left(Z^{h} \cdot H_{V}^{M-2}\right)+\sum_{t \in \mathbb{P}^{1}} \operatorname{deg} Z_{t}^{v}=2 n^{2}+2 M n l .
$$

Так как отображение $\tau$ определено вне подмножества коразмерности 2 , то оно действует на группе Pic $V$. Имеем

$$
\tau^{*} K_{V}=K_{V}, \quad \tau^{*} F=\sigma^{-1}(\sigma(F))-F=M H_{V}-F=-M K_{V}-F .
$$

Из предложения 2 и равенств $(2),(3)$ получаем, что одно из отображений $\chi, \chi \circ \tau$ имеет максимальную особенность $\nu$ такую, что centre $(\nu) \subset F=F_{t}$ и

$$
\operatorname{deg} Z_{t}^{v}<\frac{2 M n e(\nu)}{\nu\left(F_{t}\right)}+2 n^{2}-\left(Z^{h} \cdot H_{V}^{M-2}\right) .
$$

Бирациональная жесткость $V$ будет доказана, если мы приведем к противоречию предположение о существовании такой максимальной особенности. Это будет сделано в $\S 5$. В $\S 4$ получаются необходимые для дальнейшего оценки кратностей циклов коразмерности 2 на $V$.

Пусть теперь $\chi$ - бирациональный автоморфизм $V$. Положим $V^{\prime}=V,\left|D^{\prime}\right|=|F|$. Тогда $c\left(D^{\prime}, V^{\prime}\right)=0$. Если мы докажем, что $\chi$ не имеет максимальных особенностей, то из предыдущих рассуждений следует, что либо $c\left(\chi_{*}^{-1}(F), V\right)=0$, либо $c\left((\chi \circ \tau)_{*}^{-1}(F), V\right)=0$, т.е. одно из отображений $\chi, \chi \circ \tau$ является послойным. Из основного результата работы [9] теперь следует, что группа Bir $V /$ Aut $V$ порождается $\tau$. Но для общего многообразия $V$ группа Aut $V$ тривиальна, и мы получаем второе утверждение теоремы. 


\section{§4. Оценки кратностей циклов}

Во всех леммах этого раздела используется элементарная теория пересечений (см., например, [10]). Всюду в дальнейшем $F$ будет обозначать слой проекции $\pi$. Кратность цикла $Z$ вдоль многообразия $Y$ обозначим через mult $_{Y} Z$.

ЛЕмма 1. Пусть $p$ - гладкая точка регулярной гиперповерхности $F u$ $Z \subset F-$ эффективный неприводимый чикл коразмерности 2. Тогда при $M \geqslant 5$ верно неравенство

$$
\frac{\operatorname{mult}_{p} Z}{\operatorname{deg} Z} \leqslant \max \left\{\frac{3}{M}, \frac{8}{3(M-1)}\right\}
$$

ДоказАТЕЛьство. Пусть $T_{p} F-$ касательная гиперплоскость к $F$ в $p$.

Рассмотрим два случая.

1) $Z$ не содержится в $T_{p} F \cap F=T$. Тогда $(Z \circ T)$ - эффективный цикл коразмерности 3 на $F$ и по доказанному в [9]

$$
\frac{5}{2 M} \geqslant \frac{1}{2} \frac{\operatorname{mult}_{p}(Z \circ T)}{\operatorname{deg}(Z \circ T)} \geqslant \frac{\operatorname{mult}_{p} Z}{\operatorname{deg} Z} .
$$

2) $Z \subset T$. Пусть $Q$ - проективизация квадратичного касательного конуса к $T$ в точке $p$ и $S=Q \cap T$. Предположим сначала, что цикл $Z$ не является компонентой $S$. Рассмотрим линейные системы $\Lambda_{k}=|k L-(k+1) E|, k=2, \ldots, M-1$, где $L-$ гиперплоское сечение $T$, а $E$ - исключительный дивизор раздутия точки $p$ (см. $[3],[9])$. Так как $p$ - регулярная точка $F$, то коразмерность базисного множества линейной системы $\Lambda_{k}$ в $T$ не меньше $k-1$ (см. [9]), следовательно, для общих дивизоров $D_{k} \in \Lambda_{k}$ одномерный цикл $Z^{*}=\left(Z \circ S \circ D_{4} \circ \cdots \circ D_{M-2}\right)$ эффективен. Имеем

$$
1 \geqslant \frac{\operatorname{mult}_{p} Z^{*}}{\operatorname{deg} Z^{*}} \geqslant \frac{\operatorname{mult}_{p} Z}{\operatorname{deg} Z} \cdot \frac{3}{2} \cdot \frac{5}{4} \cdots \frac{M-1}{M-2}=\frac{3(M-1) \operatorname{mult}_{p} Z}{8 \operatorname{deg} Z} .
$$

Пусть теперь $Z$ - компонента $S$ и $M \geqslant 6$. Если многообразие $T$ имеет особенность вдоль кривой $C$, то $C$ содержит особую точку $F$. Однако легко показать, что пересечение $F$ с любой гиперплоскостью, проходящей через регулярную особую точку, имеет в этой точке изолированную особенность. Таким образом, по теореме Гротендика (см. [11]) точка $p$ является факториальной особой точкой многообразия $T$, поэтому $Z$ высекается в $T$ компонентой конуса $Q$ и выполняется равенство

$$
\frac{\operatorname{mult}_{p} Z}{\operatorname{deg} Z}=\frac{3}{M}
$$

т.е. утверждение леммы верно.

Пусть, наконец, $M=5$ и $Z$ является компонентой $S$. Если $Z=S$, то mult ${ }_{p} Z=6$, $\operatorname{deg} Z=10$ и утверждение леммы выполнено, поэтому в дальнейшем будем предполагать, что $Z \neq S$. Обозначим mult $_{p} Z=k, \operatorname{deg} Z=d, k<6, d<10$. Для общего дивизора $D_{3} \in \Lambda_{3}$ цикл $Z^{\prime}=\left(Z \circ D_{3}\right)$ эффективен и

$$
1 \geqslant \frac{\operatorname{mult}_{p} Z^{\prime}}{\operatorname{deg} Z^{\prime}} \geqslant \frac{4 \operatorname{mult}_{p} Z}{3 \operatorname{deg} Z}
$$

откуда следует, что $k \leqslant \frac{3}{4} d$. 
Так как гиперповерхность $F$ регулярна, то для любой точки $q \in F$ и для любого цикла $W \subset F$ коразмерности 2 выполнено неравенство

$$
\frac{\operatorname{mult}_{q} W}{\operatorname{deg} W} \leqslant \frac{4}{5}
$$

(см. [3]), в частности $F$ не содержит двумерных плоскостей.

Докажем теперь, что выполнено неравенство

$$
\frac{\text { mult }_{p} Z}{\operatorname{deg} Z} \leqslant \frac{2}{3} \text {. }
$$

Если $k \leqslant d-3$, то

$$
\frac{k}{d} \leqslant \frac{d-3}{d} \leqslant \frac{2}{3}
$$

При $k \geqslant d-2$ имеются только две возможности, для которых $2 / 3<k / d \leqslant 3 / 4$ : $k=3, d=4$ и $k=5, d=7$. Докажем, что они не реализуются.

Рассмотрим два случая.

а) Конус $Q$ неприводим и приведен. Если $k=3, d=4$, то $Z$ содержится в трехмерной гиперплоскости $H$, т.е. $Z \subset H \cap Q$, что невозможно. Если $k=5, d=7$, то $S=Z \cup W$, где mult ${ }_{p} W=1, \operatorname{deg} W=3$. Так как $F$ не содержит плоскостей, то цикл $W$ неприводим. Но в этом случае $W$ не может содержаться в $Q$.

б) Конус $Q$ распадается на две гиперплоскости или не приведен. Тогда $Z$ является компонентой поверхности пятой степени в $\mathbb{P}^{3}$, поэтому $\operatorname{deg} Z \leqslant 5$. Случай $d=4$ невозможен, так как $F$ не содержит плоскостей.

Лемма доказана.

ЛЕмма 2. Если $p$ - регулярная особая точка $F, Z$ - әффективный иикл коразмерности 2 на $V$, не содержащийся в $F$, то

$$
\frac{\operatorname{mult}_{p} Z}{\operatorname{deg} Z} \leqslant \frac{2}{M} .
$$

ДокАЗАТЕЛЬСтво. По доказанному в [3] имеем

$$
\frac{4}{M} \geqslant \frac{\operatorname{mult}_{p}(Z \circ F)}{\operatorname{deg}(Z \circ F)} \geqslant 2 \frac{\operatorname{mult}_{p} Z}{\operatorname{deg} Z} .
$$

ЛЕмма 3. Для любого простого дивизора $Y \subset F$ и регулярной неособой точки $p \in F$ верно неравенство

$$
\frac{\operatorname{mult}_{p} Y}{\operatorname{deg} Y} \leqslant \frac{2}{M} .
$$

ЛЕмма 4. Если р-регулярная особая точка $F, Y$ - дивизор на $F$, то

$$
\frac{\operatorname{mult}_{p} Y}{\operatorname{deg} Y} \leqslant \frac{3}{M}
$$

ЛЕмма 5. Пусть $p$ - регулярная особая точка $F, Y$ - дивизор на $F, \tilde{Y}$ его собственный прообраз при раздутии точки $p, x$ - точка исключительного дивизора раздутия. Тогда выполняется неравенство

$$
\frac{\operatorname{mult}_{x} \tilde{Y}}{\operatorname{deg} Y} \leqslant \frac{2}{M} .
$$

Доказательство лемм 3-5 см. в [3]. 
ОПРЕДЕЛЕНИЕ 6. Горизонтальной прямой на $V$ назовем одномерньй цикл, имеющий вид $\sigma^{-1}(x)$ для некоторой точки $x \in \mathbb{P}^{M}$.

Лемма 6. Пусть $L$ - горизонтальная прямая на $V$. Тогда верно одно из двух утверәсдений:

1) $\operatorname{mult}_{L} Z^{h} \leqslant \frac{1}{2} \operatorname{deg} Z^{h}$

2) $\left(Z^{h} \cdot H_{V}^{M-2}\right) \geqslant \operatorname{deg} Z^{h}$.

ДокАЗАтЕльство. Пусть $p \in L-$ произвольная точка, $F_{p}$ - слой, содержаший $p$, и $T_{p} F_{p}-$ касательная гиперплоскость к $F_{p}$ в $p$. Если цикл $Z_{p}^{h}=\left(Z^{h} \circ F_{p}\right)$ не содержится в $T_{p}=T_{p} F_{p} \cap F_{p}$, то

$$
\operatorname{mult}_{L} Z^{h} \leqslant \operatorname{mult}_{p} Z_{p}^{h} \leqslant \frac{1}{2} \operatorname{mult}_{p}\left(Z_{p}^{h} \circ T_{p}\right) \leqslant \frac{1}{2} \operatorname{deg} Z^{h} .
$$

Поэтому либо верно первое утверждение, либо цикл $Z^{h}$ содержится в многообразии $T=\bigcup_{p \in L} T_{p} F_{p} ; T$ задается в $\mathbb{P}^{1} \times \mathbb{P}^{M}$ уравнением $Q u^{2}+R u v+S v^{2}=0$, где $Q, R, S$ - линейные формы.

Рассмотрим бирациональную инволюцию $\widetilde{\tau}: T \rightarrow T$, которая переставляет точки в слоях проекции $\tilde{\sigma}: T \rightarrow \mathbb{P}^{M}$. Пусть $K$ - подмногообразие в $\mathbb{P}^{M}$, определенное уравнениями $Q=R=S=0$. Легко видеть, что $K$ - касательная гиперплоскость к $W$ в точке $\widetilde{\sigma}(L)$. Отображение $\widetilde{\tau}$ является бирегулярной инволюцией вне $\widetilde{\sigma}^{-1}(K)$. Пусть $H_{T}=\left.H\right|_{T}$ и $F_{T}=\left.G\right|_{T}$ - образующие группы $\operatorname{Pic~} T$. Так как линейная система $\left|F_{T}\right|$ не имеет базисных точек и $\widetilde{\tau}\left(\left|F_{T}\right|\right)=\left|H_{T}-F_{T}\right|$, то базисное множество линейной системы $\left|H_{T}-F_{T}\right|$ содержится в $\widetilde{\sigma}^{-1}(K)$. Поскольку $W$ гладко и неприводимо, $K$ не содержится в $W$ и размерность $K$ равна $M-3$, поэтому $\widetilde{\sigma}^{-1}(K)$ не содержится в $V$ и $\operatorname{dim}\left(V \cap \widetilde{\sigma}^{-1}(K)\right) \leqslant M-3$. Отсюда следует, что неприводимые компоненты цикла $Z^{h}$ не содержатся в базисном множестве линейной системы $\left|H_{T}-F_{T}\right|$, значит, для общего дивизора $D \in\left|H_{T}-F_{T}\right|$ цикл $\left(Z^{h} \circ D\right)$ эффективен. Так как линейная система $\left|H_{T}\right|$ свободна, то

$$
\begin{aligned}
\left(Z^{h} \cdot H_{V}^{M-2}\right) & =\left(Z^{h} \cdot H_{T}^{M-2}\right)=\left(Z^{h} \cdot H_{T}^{M-3} \cdot\left(H_{T}-F_{T}\right)\right)+\left(Z^{h} \cdot H_{T}^{M-3} \cdot F_{T}\right) \\
& \geqslant\left(Z^{h} \cdot H_{T}^{M-3} \cdot F_{T}\right)=\operatorname{deg} Z^{h} .
\end{aligned}
$$

Лемма доказана.

\section{§5. Исключение максимальной особенности}

Пусть $\varphi_{i, i-1}: V_{i} \rightarrow V_{i-1}, i=1, \ldots, K,-$ разрешение дискретного нормирования $\nu$ (подробности см. в [2], [8]), где $\varphi_{i, i-1}-$ раздутие с центром $B_{i-1} \subset V_{i-1}$ и исключительным дивизором $E_{i} \subset V_{i}$, причем первые $L \leqslant K$ раздутий соответствуют циклам $B_{i-1}$ коразмерности $\geqslant 3$, а первые $N \leqslant L-$ циклам $B_{i-1} \subset F^{i-1}$ (верхний индекс обозначает собственный прообраз относительно соответствующего раздутия). На множестве индексов $\{1, \ldots, K\}$ введем структуру ориентированного графа: $j$ и $i$ соединяются стрелкой, если $j>i$ и $B_{j-1} \subset E_{i}^{j-1}$. Пусть $p_{i}-$ число путей в этом графе, ведуших из вершины $K$ в вершину $i$. Обозначим

$$
\begin{gathered}
\Sigma_{l}=\sum_{i=1}^{L} p_{i}, \quad \Sigma_{u}=\sum_{i=L+1}^{K} p_{i}, \quad \Sigma_{f}=\sum_{i=1}^{N} p_{i}, \quad d_{h}=\operatorname{deg} Z^{h}, \quad d_{v}=\operatorname{deg} Z_{t}^{v}, \\
m_{i}^{h}=\operatorname{mult}_{B_{i-1}}\left(Z^{h}\right)^{i-1}, \quad m_{i}^{v}=\operatorname{mult}_{B_{i-1}}\left(Z_{t}^{v}\right)^{i-1}, \\
m_{i}^{h} \leqslant m_{1}^{h}=m^{h}, \quad m_{i}^{v} \leqslant m_{1}^{v}=m^{v} .
\end{gathered}
$$


Так же как в [2], мы получаем неравенство

$$
\sum_{i=1}^{L} p_{i} m_{i}^{h}+\sum_{i=1}^{N} p_{i} m_{i}^{v} \geqslant 4 n^{2} \Sigma_{l}+4 n e .
$$

Рассмотрим два случая.

1) $B_{0}=\operatorname{centre}(\nu)$ - особая точка $F$. Положим $\Sigma_{f}^{\prime}=2 p_{1}+\sum_{i=2}^{N} p_{i}$. Тогда $\Sigma_{f}^{\prime} \leqslant 2 \Sigma_{l}, \nu(F) \geqslant \Sigma_{f}^{\prime}$.

В силу лемм $2,4,5$ имеем

$$
m^{h} \leqslant \frac{2}{M} d_{h}=2 n^{2}, \quad m_{1}^{v} \leqslant \frac{3}{M} d_{v}<\frac{4}{M} d_{v}, \quad m_{i}^{v} \leqslant \frac{2}{M} d_{v}, \quad i \geqslant 2 .
$$

Учитывая неравенство

$$
d_{v}<2 n^{2}+\frac{2 M n e}{\nu(F)} \leqslant 2 n^{2}+\frac{2 M n e}{\Sigma_{f}^{\prime}}
$$

получаем

$$
\begin{gathered}
\sum_{i=1}^{L} p_{i} m_{i}^{h}+\sum_{i=1}^{N} p_{i} m_{i}^{v} \leqslant m^{h} \Sigma_{l}+p_{1} m_{1}^{v}+\sum_{i=2}^{N} p_{i} m_{i}^{v}<2 n^{2} \Sigma_{l}+\frac{4 n^{2}}{M} \Sigma_{f}^{\prime}+4 n e \\
\frac{4 n^{2}}{M} \Sigma_{f}^{\prime}>2 n^{2} \Sigma_{l} .
\end{gathered}
$$

Противоречие при $M \geqslant 4$.

2) Общая точка цикла $B_{0}=\operatorname{centre}(\nu)$ неособа на $F$. В этом случае имеем неравенство

$$
m^{h} \Sigma_{l}+m^{v} \Sigma_{f} \geqslant 4 n^{2} \Sigma_{l}+4 n e .
$$

При $M \geqslant 5$, используя леммы 1 и 3 , получаем

$$
m^{h} \leqslant \max \left\{3 n^{2}, \frac{8 M}{3(M-1)} n^{2}\right\}, \quad m^{v} \leqslant \frac{2}{M} d_{v} .
$$

Так как

$$
d_{v}<\frac{2 M n e}{\nu(F)}+2 n^{2}-\left(Z^{h} \cdot H_{V}^{M-2}\right)
$$

и $\nu(F) \geqslant \Sigma_{f}, \Sigma_{f} \leqslant \Sigma_{l}$, то из неравенства (4) $\left(Z^{h} \cdot H_{V}^{M-2}\right)<0$ при $M \geqslant 6$, что невозможно, поскольку линейная система $\left|H_{V}\right|$ свободна, а цикл $Z^{h}$ эффективен. При $M=5$ получаем

$$
\left(Z^{h} \cdot H_{V}^{3}\right) \leqslant \frac{n^{2}}{3} .
$$

При $M=4$, воспользовавшись неравенством $m^{h} \leqslant d_{h}$, получим оценку

$$
\left(Z^{h} \cdot H_{V}^{2}\right) \leqslant 2 n^{2} .
$$

Итак, основная теорема доказана при $M \geqslant 6$, а при $M=4,5$ реализуется только первьй случай леммы 6.

Пусть $p \in B_{0}-$ произвольная точка и $H_{p}$ - элемент линейной системы $\left|H_{V}\right|$, содержащий $p$. Цикл $H_{p}$ имеет вид $\sigma^{-1}(H)$, где $H$ - гиперплоскость в $\mathbb{P}^{M}$, проходящая через точку $\sigma(p)$. Пусть $\left(Z^{h} \circ H_{p}^{M-3}\right)=k L+Z_{1}$, где $L$ - горизонтальная 
прямая, проходящая через $p$, а цикл $Z_{1}$ не содержит $L$. Общий дивизор $H_{p}$ не содержит компонент цикла $Z_{1}$, поэтому верно неравенство

$$
\operatorname{mult}_{p} Z_{1} \leqslant\left(Z_{1} \cdot H_{p}\right)=\left(Z^{h} \cdot H_{V}^{M-2}\right) .
$$

В силу леммы 6 имеем

$$
\operatorname{mult}_{p} Z^{h} \leqslant k+\operatorname{mult}_{p} Z_{1} \leqslant \frac{M}{2} n^{2}+\left(Z^{h} \cdot H_{V}^{M-2}\right) .
$$

Из неравенства

$$
m^{v} \leqslant \frac{2}{M} d_{v}<\frac{4 n e}{\Sigma_{f}}+\frac{2}{M}\left(2 n^{2}-\left(Z^{h} \cdot H_{V}^{M-2}\right)\right)
$$

и неравенств (4), (7) будем иметь

$$
\left(\frac{M}{2}+\frac{4}{M}\right) n^{2}+\left(1-\frac{2}{M}\right)\left(Z^{h} \cdot H_{V}^{M-2}\right)>4 n^{2} .
$$

С помощью оценок (5) и (6) получаем противоречие при $M=4,5$.

Доказательство основной теоремы закончено.

\section{Список литературы}

1. Исковских В.А., Манин Ю.И. Трехмерные квартики и контрпримеры к проблеме Люрота // Матем. сб. 1971. Т. 86 (128). №1. С. 140-166.

2. Пухликов $A . B$. Бирациональные автоморфизмы трехмерных алгебраических многообразий с пучком поверхностей Дель Пеццо // Изв. РАН. Сер. матем. 1998. Т. 62. № 1. C. $123-164$.

3. Пухликов А. В. Бирационально жесткие расслоения Фано // Изв. РАН. Сер. матем. 2000. T. 64. №3. C. 131-150.

4. Bardelli F. Polarized mixed Hodge structures: On irrationality of threefolds via degeneration // Ann. Mat. Pura Appl. (4). 1984. V. 137. P. 287-369.

5. Pukhlikov A. V. Certain examples of birationally rigid varieties with a pencil of double quadrics // J. Math. Sci. 1999. V. 94. № 1. P. 986-995.

6. Гриненко M. М. Бирациональные автоморфизмы трехмерного двойного конуса // Матем. сб. 1998. Т. 189. № 7. С. 37-52.

7. Гриненко М. М. Бирациональные свойства пучков поверхностей Дель Пеццо степени 1 и 2 // Матем. сб. 2000. Т. 191. № 5. С. 17-38.

8. Pukhlikov A. V. Essentials of method of maximal singularities // Explicit birational geometry of 3-folds. Cambridge: Cambridge Univ. Press, 2000. P. 73-100.

9. Pukhlikov A. V. Birational automorphisms of Fano hypersurfaces // Invent. Math. 1998. V. 134. P. 401-426.

10. Фултон У. Теория пересечений. М.: Мир, 1989.

11. Call F., Lyubeznik G. A simple proof of Grothendieck's theorem on the parafactoriality of local rings // Commutative algebra: syzygies, multiplicities, and birational algebra: AMS-IMS-SIAM summer research conference on commutative algebra, held July 4-10, 1992, Mount Holyoke College, South Hadley, MA, USA / ed. W. J. Heinzer et al. Providence, RI: Amer. Math. Soc., 1994. P. 15-18. (Contemp. Math. V. 159.)

Московский государственнњй

Поступила в редакцию университет им. М.В. Ломоносова

10.04 .2001 